\title{
3 Research Square

\section{HPV Infection and its Relationship with Expression of PIK3CA, PIK3CB in Esophageal Squamous Cells Carcinoma}

\section{Feifei Wen}

Binzhou Medical University Hospital https://orcid.org/0000-0002-2016-8287

\section{Yangyang Li}

Binzhou Medical University Hospital

\section{Shuang He}

Binzhou Medical University Hospital

\section{Xiaoyang Xu}

Binzhou Medical University Hospital

\section{Ningjie Guo}

Binzhou Medical University Hospital

Zhenzhen Jia

Binzhou Medical University Hospital

Zhongze Cui

Binzhou Medical University Hospital

\section{Lizhen Lu}

Binzhou Medical University Hospital

Shuhua Wu (D wsh8812@126.com)

Binzhou Medical University Hospital https://orcid.org/0000-0001-9914-1601

\section{Research Article}

Keywords: Esophageal squamous cell carcinoma (ESCC), HPV, p16, PIK3CA, PIK3CB, Prognosis

Posted Date: May 13th, 2021

DOI: https://doi.org/10.21203/rs.3.rs-502500/v1

License: (c) (i) This work is licensed under a Creative Commons Attribution 4.0 International License. Read Full License 


\section{Abstract}

Background: Esophageal squamous cell carcinoma (ESCC) is a highly lethal cancer. Human papillomavirus (HPV) is currently considered as a potential risk factor for ESCC, but this assumption is still contradictory. P16INK4a ( 116 ) staining can be used as an alternative marker of HPV oncogene activity. This study was to investigate the role of HPV in esophageal cancer and to compare the relationship between HPV status and PIK3CA, PIK3CB.

Methods: Immunohistochemistry, Western blot, and RT-PCR analyses were performed to detect the expression of p16, PIK3CA, PIK3CB and p53 in 156 cases of esophageal squamous cell carcinoma. The patients were followed up by telephone or clinic.

Results: The positive rates of p16, PIK3CA, PIK3CB and p53 in esophageal squamous cell carcinoma were significantly higher than those in normal esophageal mucosa. The overexpression of p16 was closely related to tumor location, TNM stage, differentiation and lymph node metastasis. In addition, the expression of p16 was positively correlated with the expression of PIK3CA, but not with the expression of PIK3CB and p53. Survival analysis showed that p16 was a good prognostic marker, while PIK3CA and p53 were poor prognostic markers.

Conclusions: HPV infection is associated with ESCC. The imbalance of PI3K/Akt signaling pathway is closely related to HPV infection and prognosis. Detection of p16 and PIK3CA is of great significance in evaluating the prognosis and optimizing the treatment of esophageal squamous cell carcinoma.

\section{Background}

Esophageal cancer (EC) represents the eighth highest incidence of cancer worldwide $(456,000$ cases/year) and is usually diagnosed at an advanced stage, showing rapid progression and extremely poor prognosis [1, 2]. The two main histological types of EC are squamous cell carcinoma and adenocarcinomaand $90 \%$ of cases are esophageal squamous cell carcinoma (ESCC). Despite advances in medical technology, such as early screening and surgery combined with chemotherapy or radiotherapy, the prognosis of EC is still very poor [3]. Therefore, elucidating the underlying pathogenesis of EC and identifying effective biological agents are urgently needed.

Viral infections, such as Epstein Barr virus (EB virus), nasopharyngeal carcinoma, and Burkitt's lymphoma, are closely related to tumorigenesis. Studies confirmed that HPV is a risk factor for the occurrence and development of multiple malignant tumors. In particular, HPV16 and HPV18 infections are closely related to the occurrence of multiple tumors $[4,5]$. For example, cervical cancer is closely related to HPV infection. The detection of HPV infection is currently used as an important indicator for the diagnosis of cervical intraepithelial neoplasia and the judgement of cancerization [6]. HPV infection is closely related to ESCC, but its tumorigenic mechanism is not clear [7]. 
Persistent HPV infection upregulates the expression of oncoproteins, especially E6 and E7. Both proteins lead to genetic instability through the inactivation of p53 and pRB, which is a negative regulator of cyclindependent kinase inhibitor p16INK4a (p16). The inactivation of pRB results in the upregulation of p16 [8]. Therefore, p16 is usually used as an alternative biomarker for HPV infection. In more than $90 \%$ of HPVpositive oropharyngeal cancer, $\mathrm{p} 16$ was overexpressed. The immunohistochemical evidence of $\mathrm{p} 16$ overexpression has been widely used to establish the correlation between HPV carcinogenic activity in cervical cancer and dysplasia [9-10]. Several studies have shown that the immunohistochemical detection of $\mathrm{p} 16$ overexpression may serve as a surrogate marker of functionally relevant HPV infection [10-11]. This detection is simple, fast, and inexpensive. In our study, p16 expression was detected by immunohistochemistry instead of by HPV detection.

Abnormal signal transduction pathway is one of the important mechanisms underlying tumorigenesis and tumor development and has become a popular research topic in the fields of tumorigenesis, target therapy, and drug resistance. The roles of viral infection in cell signaling pathway and oncogene activation are also popular topics in viral tumorigenic mechanism research. HPV infection can activate the PI3K/AKT signal transduction pathway, upregulate PIK3CA and PIK3CB expression levels, and worsen tumors [12]. The relationship between the PI3K/AKT pathway and tumorigenesis has attracted much attention in recent years [13]. The oncogene PIK3CA is a key molecule with high mutation rate in the $\mathrm{PI} 3 \mathrm{~K} / \mathrm{AKT}$ pathway. PIK3CA mutation exists in $30 \%$ of solid tumors, is related to tumorigenesis, and affects targeted therapy [14-16]. Few studies have investigated PIK3CB. In-depth studies on the correlation between HPV infection and PIK3CA and PIK3CB mutations in EC and their effects on the occurrence, development, and prognosis of EC are also still lacking.

Therefore, studying the correlation between HPV infection and PIK3CA and PIK3CB mutations in EC is important for the detection of high-risk factors, judgment of prognosis, and prevention and treatment of EC.

\section{Materials And Methods}

\section{Clinical specimens and patients' data}

A total of 156 ESCC tissue samples at different stages were collected from the Department of Pathology of Binzhou Medical University Hospital between January 2011 and May 2013. Tissue samples were routinely fixed in $10 \%$ buffered neutral formalin. Cancer tissues were cut into wedge shapes, whereas normal tissues were cut at least $5 \mathrm{~cm}$ away from the tumor margin. All ESCC patients were clinically and pathologically proven to have not received preoperative chemotherapy or radiotherapy. All specimens were collected with the informed consent of the patients, and the Ethical Committee of Binzhou Medical University Hospital approved the protocols used in this study. Clinicopathologic classification was performed according to the National Comprehensive Cancer Network classification parameters. Demographic and clinicopathological parameters were prospectively recorded using a chart review. Patients were followed-up annually by telephone or at outpatient clinics until July 2020 or death. 


\section{Immunohistochemistry and scoring}

Before staining, paraffin-embedded tissue blocks were cut into $4 \mu \mathrm{m}$-thick sections. The sections were deparaffinized in an oven at $60^{\circ} \mathrm{C}$ for $2 \mathrm{~h}$ and then rehydrated with two and three changes of xylene and ethanol, respectively. Antigen retrieval was performed using the microwave retrieval method. Endogenous peroxidase activity was quenched through incubation with $3 \%$ hydrogen peroxide for $10 \mathrm{~min}$ at room temperature. Nonspecific binding was blocked by incubating the sections with $10 \%$ normal goat serum in PBS for 30 min at room temperature. Without washing, the sections were incubated with rabbit monoclonal antibody against human PIK3CA (1:150, Abcam, Cambridge, MA, USA), PIK3CB (1:250, Abcam, Cambridge, MA, USA), p16 (Fuzhou Maixin Biotech Co.), and p53 (Fuzhou Maixin Biotech Co.) at $4{ }^{\circ} \mathrm{C}$ overnight. The sections were then incubated with horseradish peroxidase-conjugated secondary goat anti-rabbit antibody (Abcam, San Francisco, USA) for $1 \mathrm{~h}$ at room temperature. They were washed with PBS and treated with the metal-enhanced DAB substrate kit (Thermo Scientific, USA) to visualize the antigen-antibody complex. Two researchers who were unaware of the clinicopathological status of the specimens, scored each section separately. The percentage of stained cells on each section was scored as follows: 0 (less than $5 \%), 1(5 \%-25 \%), 2(26 \%-50 \%)$, and 3 (> 50\%). Staining intensity was scored as follows: 0 (no staining), 1 (weak staining), 2 (moderate staining), and 3 (strong staining). The final score of each specimen was calculated by the multiplying stained cell score with the staining intensity score. Thus, the final score ranged from 0 to 9 . Low expression was defined as a final score of $<4$, whereas a score $\geq 4$ was determined to be high expression.

\section{Western blotting analysis}

Tissue samples were homogenized in SDS buffer containing the protease inhibitor PMSF. The homogenate on ice for $20 \mathrm{~min}$, then at $4^{\circ} \mathrm{C}$ for 30 minutes was centrifuged at $12000 \mathrm{rpm}$. Collect the supernatant and add a similar volume of $2 \times$ SDS buffer. Boil the mixture for 10 minutes and store at $-20^{\circ} \mathrm{C}$. The protein extract $(50 \mu \mathrm{g})$ was separated by SDS-PAGE, and then transferred to a polyvinylidene

fluoride membrane (Millipore, USA). The membrane was blocked with $5 \%$ skim milk in Tris buffered saline containing $0.1 \%$ Tween-20 for 90 minutes at room temperature. Then immunoblotting was used to detect PIK3CA (1:1000, Abcam), PIK3CB (1:1000, Abcam), p16 (1:500, Abcam), p53 (1:500, Abcam) and $\beta$-actin (1: 1000, Abcam). Use horseradish peroxidase secondary antibody (1:5000, Abcam) to detect protein bands and display them with enhanced chemiluminescence reagents. Each band is quantified by densitometry, and the results are expressed in terms of the relative expression of each protein in different samples.

\section{Real-time PCR analysis}

Trizol reagent (Invitrogen, China) was used to extract total RNA from fresh frozen tumor specimens and corresponding non-cancerous tissues. The content of RNA was measured by the absorbance at $260 \mathrm{~nm}$, and the purity was estimated by the ratio of absorbance at A260/280. Total RNA was reverse transcribed using PrimeScript RT kit (Takara, DRR037A, China). The real-time PCR reaction was performed on the CFX96TM real-time PCR detection system C1000 (Applied Biosystems, China). For real-time PCR 
reactions based on SYBR GREEN I, each $25 \mu \mathrm{L}$ reaction mixture contains $2 \mu \mathrm{L}$ primer pairs, $2 \mu \mathrm{L} \mathrm{cDNA}$, $12.5 \mu \mathrm{L}$ SYBR Premix Ex Taq II and $8.5 \mu \mathrm{L} \mathrm{ddH}_{2} \mathrm{O}$ to obtain a final volume of $25 \mu \mathrm{L}$.

\section{Statistical analysis}

All statistical analyses were conducted with the SPSS 22.0 software (SPSS Inc., Chicago, USA). The expression of protein and mRNA was expressed by mean \pm standard deviation. The comparison between the two groups was performed by independent sample t test. The correlation of p16 with PIK3CA, PIK3CB, p53 expression and clinical parameters was analyzed using $X$ and Fisher's exact tests. Overall survival (OS) was plotted using the Kaplan-Meier method. The analyses of prognostic factors for OS were determined using multivariate Cox proportional hazards regression method. $P$ value $\leq 0.05$ was considered statistically significant.

\section{Results}

\section{Expression levels of p16, PIK3CA, PIK3CB and p53 in ESCC samples}

Immunohistochemistry was used in human ESCC and matched adjacent non-tumor tissues to explore the expression levels of p16, PIK3CA, PIK3CB and p53. The immunohistochemical results are shown in Fig. 1. The expression levels of p16, PIK3CA and PIK3CB and p53 were positive in the cytoplasm, nuclei, and cytoplasm and nuclei of the ESCC samples, respectively. The positive ratios of p16, PIK3CA, PIK3CB, p53 and were $30.8 \%, 20.50 \%, 15.4 \%$ and $65.4 \%$, respectively. As shown in Table 1, p16, PIK3CA, PIK3CB and p53 expression levels were significantly higher in the ESCC samples than in the non-tumor tissues $(P<$ 0.05). In addition, Western blot analysis and real-time PCR results showed that $p 16$, PIK3CA, PIK3CB and p53 expression was significantly higher in the CRC samples than in the non-tumor tissues $(P<0.05)$ (Fig. 2).

Table 1

Expression of p16, PIK3CA, PIK3CBand p53 protein in esophageal squamous cell carcinoma and normal mucosa

\begin{tabular}{|llll|}
\hline group & Normal mucosa & Cancer & $P$ value \\
\hline p16 & $0(0 / 156)$ & $30.8(48 / 156)$ & $0.001^{*}$ \\
\hline PIK3CA & $2.5(4 / 156)$ & $20.5(32 / 156)$ & $0.001^{*}$ \\
\hline PIK3CB & $1.3(2 / 156)$ & $15.4(24 / 156)$ & $0.001^{*}$ \\
p53 & $1.9(3 / 156)$ & $65.4(102 / 156)$ & $0.000^{*}$ \\
\hline
\end{tabular}


*indicate statistically significant $(p<0.05)$

\section{Correlation of p16 with PIK3CA, PIK3CB, p53 and different clinicopathologic parameters}

The correlation between p16 expression levels of the ESCC samples and a set of clinicopathologic parameters, including age, gender, tumor location, TNM stage, differentiation grade, and lymph node metastasis was analyzed (Table 2). The expression of $p 16$ was significantly correlated with esophageal location, differentiation grade, TNM stage, and lymph node metastasis. The expression rate of p16 in the cervical/upper part (64.71\%) of the esophagus was significantly higher than that in the middle (30.36\%) and low $(24.10 \%)$ parts. In addition, p16 expression was higher in well-differentiated ESCC $(43.14 \%)$ than in moderately and poorly differentiated ESCCs $(27.40 \%$ and $18.75 \%$, respectively). The p 16 expression levels in stages $₫$ and $\otimes(37.5 \%)$ were higher than those in stages $\nabla$ and $\otimes(20 \%)$. The expression of $p 16$ was lower in the presence of lymph node metastasis (20.97\%) than in its absence (37.23\%). PIK3CA expression was positively correlated with $\mathrm{p} 16$ ( $r \mathrm{~s}=0.175, P=0.027)$. However, PIK3CB and p53 expression levels were not correlated with $\mathrm{p} 16$ expression. 
Table 2

Epidemiological data and statistical analysis of the correlation between the positivity of p16 and the different data analyzed

\begin{tabular}{|c|c|c|c|c|c|}
\hline \multirow[t]{3}{*}{ Parameters } & \multicolumn{2}{|c|}{ p16 expression } & \multirow{3}{*}{$\begin{array}{l}\text { Total } \\
\mathrm{N}=156\end{array}$} & \multirow[t]{3}{*}{ rvalue } & \multirow[t]{3}{*}{$P$ value } \\
\hline & Positive & Negative & & & \\
\hline & $N=48$ & $N=108$ & & & \\
\hline \multicolumn{6}{|l|}{ Age (years) } \\
\hline$<60$ & $20(42 \%)$ & $33(31 \%)$ & $53(34 \%)$ & 0.108 & 0.176 \\
\hline$\geq 60$ & $28(58 \%)$ & $75(69 \%)$ & $103(66 \%)$ & & \\
\hline \multicolumn{6}{|l|}{ Gender } \\
\hline Male & $29(60 \%)$ & $81(75 \%)$ & $110(71 \%)$ & 0.146 & 0.086 \\
\hline Female & $19(40 \%)$ & $27(25 \%)$ & $46(29 \%)$ & & \\
\hline \multicolumn{6}{|l|}{ Tumor location } \\
\hline Cervical/Upper & $11(23 \%)$ & $6(5 \%)$ & $17(11 \%)$ & 0.256 & $0.004^{*}$ \\
\hline Middle & $17(35 \%)$ & $39(36 \%)$ & $56(36 \%)$ & & \\
\hline Low & $20(42 \%)$ & $63(59)$ & $83(53 \%)$ & & \\
\hline \multicolumn{6}{|l|}{ TNM stage } \\
\hline I-II & $36(75 \%)$ & $60(56 \%)$ & $96(62 \%)$ & 0.169 & $0.047^{\star}$ \\
\hline III-IV & $12(25 \%)$ & $48(44 \%)$ & $60(38 \%)$ & & \\
\hline \multicolumn{6}{|l|}{ Differentiation grade } \\
\hline Well & $22(46 \%)$ & $29(27 \%)$ & $51(33 \%)$ & -0.196 & $0.045^{*}$ \\
\hline Moderate & $20(41.5 \%)$ & $53(49 \%)$ & $73(47 \%)$ & & \\
\hline Poor & $6(12.5)$ & $26(24 \%)$ & $32(20 \%)$ & & \\
\hline \multicolumn{6}{|l|}{ Depth of invasion } \\
\hline Mucosa and submucosa & $10(21 \%)$ & $16(15 \%)$ & $26(17 \%)$ & 0.123 & 0.303 \\
\hline Myometrium & $15(31 \%)$ & $26(24 \%)$ & $41(26 \%)$ & & \\
\hline Adventitia & $23(48 \%)$ & $66(61 \%)$ & $89(57 \%)$ & & \\
\hline \multicolumn{6}{|l|}{ Lymph node metastasis } \\
\hline Yes & $13(27 \%)$ & $49(45 \%)$ & $62(40 \%)$ & -0.170 & $0.035^{*}$ \\
\hline No & $35(73 \%)$ & $59(55 \%)$ & $94(60 \%)$ & & \\
\hline
\end{tabular}




\begin{tabular}{|c|c|c|c|c|c|}
\hline \multirow[t]{3}{*}{ Parameters } & \multicolumn{2}{|c|}{ p16 expression } & \multirow{3}{*}{$\begin{array}{l}\text { Total } \\
\mathrm{N}=156\end{array}$} & \multirow[t]{3}{*}{ r value } & \multirow[t]{3}{*}{$P$ value } \\
\hline & Positive & Negative & & & \\
\hline & $N=48$ & $N=108$ & & & \\
\hline \multicolumn{6}{|l|}{ PIK3CA } \\
\hline Positive & $15(31 \%)$ & $17(16 \%)$ & $32(21 \%)$ & 0.175 & $0.027^{*}$ \\
\hline Negative & $33(69 \%)$ & $91(84 \%)$ & 124(79\%) & & \\
\hline \multicolumn{6}{|l|}{ РІКЗСВ } \\
\hline Positive & $11(23 \%)$ & $13(12 \%)$ & $24(15 \%)$ & 0.138 & 0.138 \\
\hline Negative & $37(77 \%)$ & $95(88 \%)$ & $132(85 \%)$ & & \\
\hline \multicolumn{6}{|l|}{ P53 } \\
\hline Positive & $30(62.5 \%)$ & $72(67 \%)$ & $102(65 \%)$ & 0.040 & 0.614 \\
\hline Negative & $18(37.5 \%)$ & $36(33 \%)$ & $54(35 \%)$ & & \\
\hline
\end{tabular}

*indicate statistically significant $(p<0.05)$

\section{Correlation between PIK3CA, PIK3CB, p53 and p16 expression levels and patient OS}

The median survival time of 156 patients was 44 months, and their 5 -year survival rate was $28.2 \%$. The 5 year survival rates of patients with positive and negative p16 expression levels were $39.6 \%$ and $23.1 \%$, respectively $(P<0.05$, Fig. 3B). Kaplan-Meier analysis (Fig. 3) revealed that p16, PIK3CA, p53, depth of invasion, TNM stage, and lymph node metastasis could be relevant predictive factors of OS. The 5-year survival rates of patients with positive and negative p16 expression levels were $39.6 \%$ and $23.1 \%$, respectively $(P<0.01$, Fig. 3A). The 5-year survival rates of patients with positive and negative PIK3CA expression levels were $18.7 \%$ and $30.6 \%$, respectively $(P<0.05$, Fig. 3B). The 5 -year survival rates of patients with positive and negative p53 expression levels were $23.5 \%$ and $37.1 \%$, respectively $(P<0.05$, Fig. 3D). Thus, p16-positive ESCC was a distinct entity with a favorable prognosis compared with p16negative ESCC. PIK3CA and p53 were markers of poor prognosis in ESCC. Cox regression analysis showed that p16 and PIK3CA expression levels, differentiation grade, and depth of invasion were independent prognostic factors of ESCC (Table 3). 
Table 3

Univariate and multivariate analysis of clinicopathologic parameters with OS by Cox proportional hazards regression

\begin{tabular}{|c|c|c|c|c|c|c|c|}
\hline \multirow[t]{2}{*}{ Parameters } & \multirow[t]{2}{*}{$\beta$} & \multirow[t]{2}{*}{ SE } & \multirow[t]{2}{*}{ Wald } & \multirow[t]{2}{*}{$\operatorname{Exp}(\beta)$} & \multicolumn{2}{|l|}{$95 \% \mathrm{Cl}$} & \multirow{2}{*}{$\begin{array}{l}P \\
\text { value }\end{array}$} \\
\hline & & & & & Lower & Upper & \\
\hline Age $(<60, \geq 60)$ & 0.219 & 0.198 & 1.221 & 1.245 & 0.844 & 1.837 & 0.269 \\
\hline Gender (male, female) & 0.325 & 0.208 & 2.453 & 1.384 & 0.922 & 2.079 & 0.117 \\
\hline Tumor location & & & 3.026 & & & & 0.220 \\
\hline $\begin{array}{l}\text { Tumor location (Cervical/Upper, } \\
\text { Middle) }\end{array}$ & 0.307 & 0.345 & 0.790 & 0.736 & 0.374 & 1.447 & 0.374 \\
\hline Tumor location (Middle, Lower) & 0.319 & 0.198 & 2.590 & 0.727 & 0.493 & 1.072 & 0.108 \\
\hline $\begin{array}{l}\text { Lymph node metastasis (yes, } \\
\text { no) }\end{array}$ & 1.194 & 1.073 & 1.237 & 0.303 & 0.037 & 2.484 & 0.266 \\
\hline Differentiation grade & & & 6.747 & & & & $0.034^{\star}$ \\
\hline $\begin{array}{l}\text { Differentiation grade (Well, } \\
\text { Moderate) }\end{array}$ & 0.101 & 0.289 & 0.123 & 0.903 & 0.513 & 1.592 & 0.725 \\
\hline $\begin{array}{l}\text { Differentiation grade (Moderate, } \\
\text { Poor) }\end{array}$ & 0.539 & 0.235 & 5.271 & 0.583 & 0.368 & 0.924 & $0.022^{*}$ \\
\hline Depth of invasion & & & 12.811 & & & & $0.002^{*}$ \\
\hline $\begin{array}{l}\text { Depth of invasion (Mucosa and } \\
\text { submucosa, Myometrium) }\end{array}$ & 0.959 & 0.289 & 10.997 & 0.383 & 0.217 & 0.675 & $0.001^{*}$ \\
\hline $\begin{array}{l}\text { Depth of invasion (Myometrium, } \\
\text { Epicardial) }\end{array}$ & 0.535 & 0.237 & 5.091 & 0.586 & 0.368 & 0.932 & $0.024^{*}$ \\
\hline TNM stage (I-II, III-IV) & 0.791 & 1.071 & .546 & 2.207 & 0.271 & 18.000 & 0.460 \\
\hline p16 & -0.524 & 0.251 & 4.371 & 0.592 & 0.362 & 0.968 & $0.037^{\star}$ \\
\hline PIK3CA & 0.796 & 0.239 & 11.107 & 2.217 & 1.388 & 3.542 & $0.001^{*}$ \\
\hline PIK3CB & 0.340 & 0.255 & 1.779 & 1.405 & 0.852 & 2.315 & 0.182 \\
\hline p53 & 0.266 & 0.207 & 1.642 & 1.304 & 0.869 & 1.958 & 0.200 \\
\hline
\end{tabular}

*indicate statistically significant $(p<0.05)$

\section{Discussion}

EC is a tumor with high invasive ability. It is usually diagnosed in the late stage due to the lack of specific initial symptoms. For late-stage EC, the main treatment is surgery combined with chemotherapy $[17,18]$. 
However, the cure and survival rates of patients are still very low, and other methods are needed to help predict the survival rate and identify potential responders of a given treatment.

HPV is a kind of DNA virus with small size and no envelope. HPV DNA is often dissociated from chromosome. In malignant tumor, HPV DNA is integrated into host cell DNA, resulting in the loss of negative regulation of HPV E2 gene on HPV E6 and HPV E7 gene initiation, resulting in abnormal expression of E6 and E7 genes, leading to malignant cell proliferation. In 1982, syrjanen et al. Proposed for the first time that HPV infection may be related to the occurrence of esophageal squamous cell carcinoma[19]. In recent years, some scholars suggested that HPV could be a prognostic indicator of esophageal squamous cell carcinoma[20-22]. In head and neck cancer, patients with HPV-positive cancer have better therapeutic outcome and higher survival rate than patients with HPV-negative cancer [23-25]. A similar correlation possibly exists, because the esophagus may also be infected with HPV. HPV-infected tumors are characterized by a high expression of $\mathrm{p} 16$, which is widely considered as an alternative marker for HPV infection [10,12]. In the present study, the expression rate of p16 in ESCC was $30.8 \%$, which was significantly higher than in normal esophageal mucosa. In the correlation analysis with clinical pathological factors, p16 expression was significantly correlated with esophageal location, and the expression rate of p16 in the cervical/upper part was significantly higher than in the middle and lower parts. In addition, p16 expression was negatively correlated with the degree of differentiation, invasion depth, and lymph node metastasis of ESCC. Thus, p16 was highly expressed in patients with a high degree of differentiation, shallow invasion depth, and no lymph node metastasis. Prognosis analysis showed that the 5-year survival rate of p16-positive group was 39.6\%, whereas that of p16-negative group was $23.1 \%$. Cox regression analysis of OS and PFS showed that $\mathrm{p} 16$ status was an independent prognostic factor for OS and PFS in patients with ESCC. The present study clearly showed that p16positive tumor was related to high differentiation, TNM stage, and lymph node metastasis. These three differences may have enhanced the prognosis in patients with p16-positive tumor. Another hypothesis was that HPV infection leads to the high expression of tumor protein E6/E7, which weakens the functions of p53 and RB proteins and promotes genome rearrangement and DNA rearrangement is theoretically more sensitive to radiotherapy and chemotherapy [26-27]. This hypothesis explains the indications of increased survival rate in patients with HPV-positive tumors.

As one of the important signal transduction pathways in cells, the PI3K/Akt signaling pathway is closely related to the occurrence and development of various human tumors. Its abnormal activation promotes the normal development of cells to cancer cells. An abnormal PI3K/Akt signal transduction is present in many human malignant tumors [28-31]. As a catalytic subunit of PI3K, PI3Kp110 has four members, namely, P110a, P110ß, P1108and P110y. P110a and P110 3 , which are encoded by PIK3CA and PIK3CB, respectively, are widely found in cells. They can regulate DNA synthesis and promote normal cell development with normal expression; moreover, they are closely related to the occurrence and development of tumors with abnormal expression [32]. The mutation of PIK3CA gene can increase the expression of p110a, which receives the EGF signal. In addition, p110a is activated by Ras protein, phosphorylates downstream Akt proteinand continuously activates the PI3K/Akt signal pathway, leading to cell overproliferation. The role of $\mathrm{p} 110 \beta$ in tumorigenesis and its mechanism are unclear. In the present 
study, the positive rates of PIK3CA and PIK3CB in ESCC were significantly higher than those in the normal mucosa, suggesting the abnormal PIK3CA and PIK3CB expression levels in ESCC. High PIK3CA and PIK3CB expression levels were related to the malignant potential of EC. PIK3CA overexpression resulted in the abnormal activation of the PI3K/Akt pathway. This abnormal activation could promote the growth and proliferation of tumor cells, inhibit apoptosis, promote invasion and metastasis, regulate endothelial growth and angiogenesisand affect the efficacy of chemotherapy by catalyzing a series of protein phosphorylation.

The expression of HPV E6/E7 oncoprotein induces the carcinogenesis of HPV-transformed cells, changing various cell and molecular events by activating the PI3K/Akt signaling pathway. Xie $J$ et al. demonstrated that ALA-PDT treatment inhibits the proliferation of HeLa cells in a dose-dependent manner and effectively reduces HPV viral load via autophagy and apoptosis by regulating the Ras/Raf/MEK/ERK and PI3K/AKT/mTOR pathways [33]. Madera D et al. suggested that inhibiting PIK3CA expression and HPV can slow down the further growth of OSCC [34]. In our study, p16 expression was positively correlated with PIK3CA expression but was not correlated with PIK3CB expression. We believe that the $\mathrm{PI} 3 \mathrm{~K} / \mathrm{Akt} / \mathrm{mTOR}$ signaling pathway in HPV-infected cells was activated by mutations in pathway components and the activation of upstream signaling molecules. Activating this pathway contributes to the dysregulation of proliferation and changes in anti-apoptosis and metabolic characteristics and ultimately leads to the malignant transformation of infected cells. This signaling pathway may be a huge therapeutic opportunity and practical challenge for the treatment of HPV-induced cancer. The PI3K/Akt pathway plays a key role in many HPV-related human cancersand the HPV infection expressed in E6/E7 activates the $\mathrm{PI} 3 \mathrm{~K} / \mathrm{Akt}$ signaling pathway by changing various cell and molecular events that lead to cancer.

The differential expression of tumor suppressor protein p53 is one of the most common abnormal expressions in cancer. Mutation is mainly related to cell invasion, metastasisand late-stage tumor. The expression of HPV E6/E7 oncoprotein can induce the transformation of HPV transformed cells, which leads to the degradation and instability of p53 and pRB. In this study, the positive expression rate of p53 protein in esophageal squamous cell carcinoma was $41.1 \%$, which was significantly higher than that in normal tissuesand expression of p53 protein was related to the poor prognosis of ESCC. As a tumor suppressor gene, wild-type p53 can regulate cell cycle and avoid cell carcinogenesis. After p53 gene mutates, it loses its regulatory effect on cell growth, apoptosisand DNA repair and increases the tumor's adaptability to hypoxic microenvironment due to the change in its spatial conformation $[35,36]$. However, in the present study, no correlation was found between p53 and p16 expression levels, possibly due to the insufficient number of samples.

In conclusion, this study confirmed that HPV infection is associated with high differentiation, TNM stage and lymph node metastasis. HPV infection is a good prognostic indicator of esophageal squamous cell carcinoma. At the same time, our study showed that the increased expression levels of PIK3CA and PIK3CB were related to the occurrence of ESCC. The imbalance of PI3K/Akt signaling pathway is closely related to HPV infection and prognosis. In ESCC, PIK3CA and p53 are markers of poor prognosis. The 
combined detection of PIK3CA and p16 is of great significance for the prognosis evaluation and treatment optimization of esophageal squamous cell carcinoma.

\section{Abbreviations}

EC: Esophageal cancer, ESCC: esophageal squamous cell carcinoma, HPV: human papilloma virus, PI3K: phosphatidylinositol-3-kinase, PCR: Polymerase Chain Reaction, mRNA: messenger ribonucleic acid, OS: Overall survival.

\section{Declarations}

\section{Authors' contributions}

Feifei Wen and Shuhua Wu conceived the idea of the study, Xiaoyang Xu, Ningjie Guo, Zhenzhen Jia and Lizhen Lu performed the research, Feifei Wen, Shuhua Wu, Yangyang Li, Shuang He and Zhongze Cui analysed the data, Feifei Wen and Shuhua Wu interpreted the results, Feifei Wen wrote the paper, all authors discussed the results and revised the manuscript.

\section{Funding}

This work was supported by the National natural science foundation, China (No: 50012301142), the science and technology project of Binzhou Medical University (No: BY2015KJ04).

\section{Availability of data and materials}

All the generated data in this review are included in the manuscript.

\section{Ethics approval and consent to participate}

The experimental protocol was established, according to the ethical guidelines of the Helsinki Declaration and was approved by the Human Ethics Committee of Binzhou Medical University Hospital Written informed consent was obtained from individual or guardian participants.

\section{Consent for publication}

Not applicable.

\section{Competing interests}

The authors declare that they have no competing interests.

\section{Author details}

Department of Pathology, Binzhou Medical University Hospital, Binzhou, Shandong Province 256603, China. 
No.

\section{References}

1. Bognár L, Hegedűs I, Bellyei S, et al. Prognostic role of HPV infection in esophageal squamous cell carcinoma. Infect Agent Cancer. 2018;29:13:38.

2. Ferlay J, Soerjomataram I, Dikshit R, et al. Cancer incidence and mortality worldwide: sources, methods and major patterns in GLOBOCAN 2012. I Int J Cancer. 2015;136:E359-86.

3. Jeong DY, Lee KS, Choy JY, et al. Surgically Resected Esophageal Squamous Cell Carcinoma: Patient Survival and Clinicopathological Prognostic Factors. Sci Rep. 2020;10:50774.

4. Lu X, Zhou Y, Meng J, et al. Epigenetic age acceleration of cervical squamous cell carcinoma converged to human papillomavirus 16/18 expression, immunoactivation, and favourable prognosis. Clin Epigenetics. 2020;12:23.

5. Stankiewicz Karita HC, Magaret A, Huang ML, et al. Quantitative Oral HPV16 and HPV18 Detection in Persons Attending Dental Clinics. Sex Transm Dis. 2020;47:100-4.

6. Peeters E, Wentzensen N, Bergeron C, et al. Meta-analysis of the accuracy of p16 or p16/Ki-67 immunocytochemistry versus HPV testing for the detection of CIN2+/CIN3 + in triage of women with minor abnormal cytology. Cancer Cytopathol. 2019;127:169-80.

7. HEl-Zimaity,Vincenzo Di Pilato, Ringressi MN, et al. Risk factors for esophageal cancer: emphasis on infectious agents. Ann N Y Acad Sci. 2018; 1434: 319-332.

8. Li S, Hong X, Wei Z, et al. Ubiquitination of the HPV Oncoprotein E6 Is Critical for E6/E6AP-Mediated p53 Degradation. Front Microbiol. 2019;10:2483.

9. Annetmary Sabu NV, Ratna Mouli N, Tejaswini, et al. Human Papillomavirus Detection in Oropharyngeal Squamous Cell Carcinoma Using p16 Immunohistochemistry. Int J Appl Basic Med Res. 2019;9:212-6.

10. Gurín D, Slávik M, Shatokhina T, et al. Current Perspective on HPV-Associated Oropharyngeal Carcinomas and the Role of p16 as a Surrogate Marker of High-Risk HPV. Klin Onkol. 2019;32:25260.

11. Irene T, Ovestad I, Dalen E, Hansen, et al. Clinical value of fully automated p16/Ki-67 dual staining in the triage of HPV-positive women in the Norwegian Cervical Cancer Screening Program. Cancer Cytopathol. 2017;125:283-91.

12. Ruxing Xi S, Pan X, Chen, et al. HPV16 E6-E7 induces cancer stem-like cells phenotypes in esophageal squamous cell carcinoma through the activation of PI3K/Akt signaling pathway in vitro and in vivo. Oncotarget. 2016;7:57050-65.

13. Konstantia E, Tasioudi S, Sakellariou G, Levidou, et al. Immunohistochemical and molecular analysis of PI3K/AKT/mTOR pathway in esophageal carcinoma. APMIS. 2015;123:639-47. 
14. Madsen RR, Vanhaesebroeck B, Semple RK. Cancer-Associated PIK3CA Mutations in Overgrowth Disorders. Trends Mol Med. 2018;24:856-70.

15. Zhang S, Cai J, Xie W, et al. miR-202 suppresses prostate cancer growth and metastasis by targeting PIK3CA. Exp Ther Med. 2018;16:1499-504.

16. Shuhua Wu F, Wen Y, Li, et al. PIK3CA and PIK3CB silencing by RNAi reverse MDR and inhibit tumorigenic properties in human colorectal carcinoma. Tumour Biol. 2016;37:8799-809.

17. Fatehi Hassanabad A, Chehade R, Breadner D, et al. Esophageal carcinoma: Towards targeted therapies. Cell Oncol (Dordr). 2020;43:195-209.

18. Wang W-L, Wang Y-C, Lee C-T, et al. The impact of human papillomavirus infection on the survival and treatment response of patients with esophageal cancers. J Dig Dis. 2015;16:256-63.

19. Syrjanen K, Pyrhonen S, Aukee $S$, et al. Squamous cell papilloma of the esophagus: a tumour probably caused by human papilloma virus (HPV). Diagn Histopathol. 1982;5(4):291-6.

20. Bognár L, Hegedűs I, Bellyei S, et al. Prognostic role of HPV infection in esophageal squamous cell carcinoma. Infect Agent Cancer. 2018;13:38.

21. da Costa AM, Fregnani JHTG, Pastrez PRA, et al. HPV infection and p53 and p16 expression in esophageal cancer: are they prognostic factors? Infect Agent Cancer. 2017; Oct 13;12:54.

22. Kumar R, Ghosh SK, Verma AK, et al. p16 Expression as a Surrogate Marker for HPV Infection in Esophageal Squamous Cell Carcinoma Can Predict Response to Neo-Adjuvant Chemotherapy. Asian Pac J Cancer Prev. 2015;16:7161-5.

23. Qian X, Nguyen DT, Dong Y, et al. Prognostic Score Predicts Survival in HPV-Negative Head and Neck Squamous Cell Cancer Patients. Int J Biol Sci. 2019;15:1336-44.

24. Chuanhao Tang L, Lin W, Zhou, et al. CDK6 inhibits lymphoid cell infiltration and represents a prognostic marker in HPV + squamous cell carcinoma of head and neck. Chin J Cancer Res. 2019;31:901-9.

25. Jitesh B, Shewale, Maura L, Gillison. Dynamic factors affecting HPV-attributable fraction for head and neck cancers. Curr Opin Virol. 2019;39:33-40.

26. Cao F, Zhang W, Zhang F, et al. Prognostic significance of high-risk human papillomavirus and p16(INK4A) in patients with esophageal squamous cell carcinoma. Int J Clin Exp Med. 2014;7:34308.

27. Saloua Kahla L, Kochbati M, Maalej, et al. Situation of HPV16 E2 gene status during radiotherapy treatment of cervical carcinoma. Asian Pac J Cancer Prev. 2014;15:2869-73.

28. Zhu Q, Hao A-LZ. Hu, et al. Acylglycerol kinase promotes tumour growth and metastasis via activating the PI3K/AKT/GSK3 $\beta$ signalling pathway in renal cell carcinoma. J Hematol Oncol. 2020;13:2.

29. Chuangyu Wen $H$, Wang $X$, Wu, et al. ROS-mediated inactivation of the PI3K/AKT pathway is involved in the antigastric cancer effects of thioredoxin reductase- 1 inhibitor chaetocin. Cell Death Dis. 2019;10:809. 
30. Li D, Ji H, Niu X, et al. Tumor-associated macrophages secrete CC-chemokine ligand 2 and induce tamoxifen resistance by activating PI3K/Akt/mTOR in breast cancer. Cancer Sci. 2020;111:47-58.

31. Carlos Eduardo Perez-Juarez. Arechavaleta-Velasco F, Zeferino-Toquero M, et al. Inhibition of $\mathrm{PI} 3 \mathrm{~K} / \mathrm{AKT} / \mathrm{mTOR}$ and MAPK signaling pathways decreases progranulin expression in ovarian clear cell carcinoma (OCCC) cell line: a potential biomarker for therapy response to signaling pathway inhibitors. Med Oncol. 2019; 37: 4.

32. Jung K, Kang H, Mehra R. Targeting phosphoinositide 3-kinase (PI3K) in head and neck squamous cell carcinoma (HNSCC). Cancers Head Neck. 2018;3:3.

33. Jiajia Xie S, Wang Z, Li, et al. 5-aminolevulinic acid photodynamic therapy reduces HPV viral load via autophagy and apoptosis by modulating Ras/Raf/MEK/ERK and PI3K/AKT pathways in HeLa cells. J Photochem Photobiol B. 2019;194:46-55.

34. Dmitri Madera L, Vitale-Cross D, Martin, et al. Prevention of tumor growth driven by PIK3CA and HPV oncogenes by targeting mTOR signaling with metformin in oral squamous carcinomas expressing OCT3. Cancer Prev Res (Phila). 2015;8:197-207.

35. Wang P, Guan D, Zhang XP, et al. Modeling the regulation of p53 activation by HIF-1 upon hypoxia. FEBS Lett. 2019;593:2596-611.

36. Zhang Y, Han CY, Duan FG, et al. p53 sensitizes chemoresistant non-small cell lung cancer via elevation of reactive oxygen species and suppression of EGFR/PI3K/AKT signaling. Cancer Cell Int. 2019;19:188.

\section{Figures}



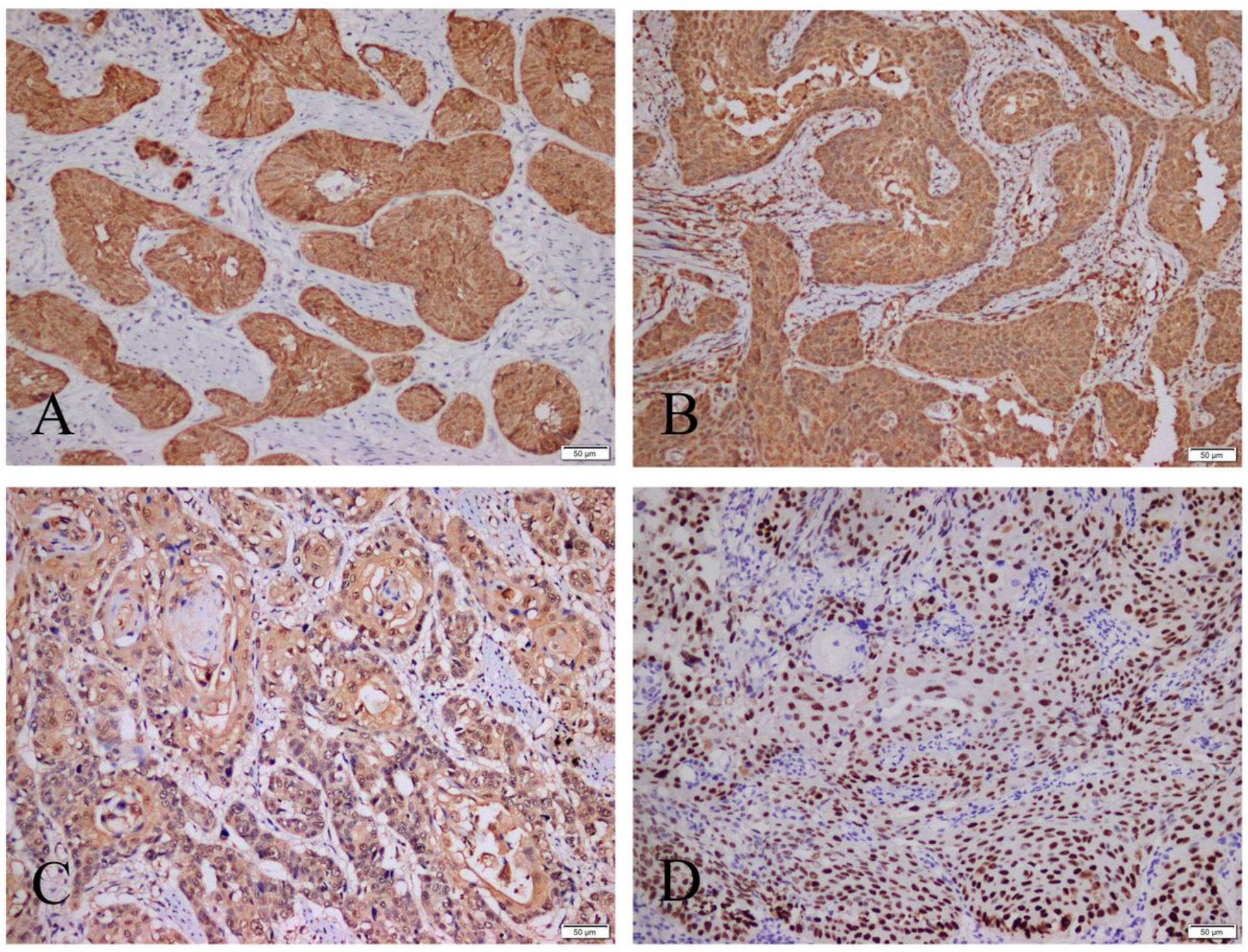

Figure 1

Immunohistochemical staining of p16, PIK3CA, PIK3CB and p53 protein expressions in esophageal squamous cell carcinomas tissues. High-power view (Original magnification $\times 200$ ) shows strong staining for p16 in the cytoplasm and nucleus of cancer cells (A), PIK3CA and PIK3CB in the of cancer cells (B, PIK3CA, C, PIK3CB), p53 in the nucleus of cancer cells (D), . 
A

B
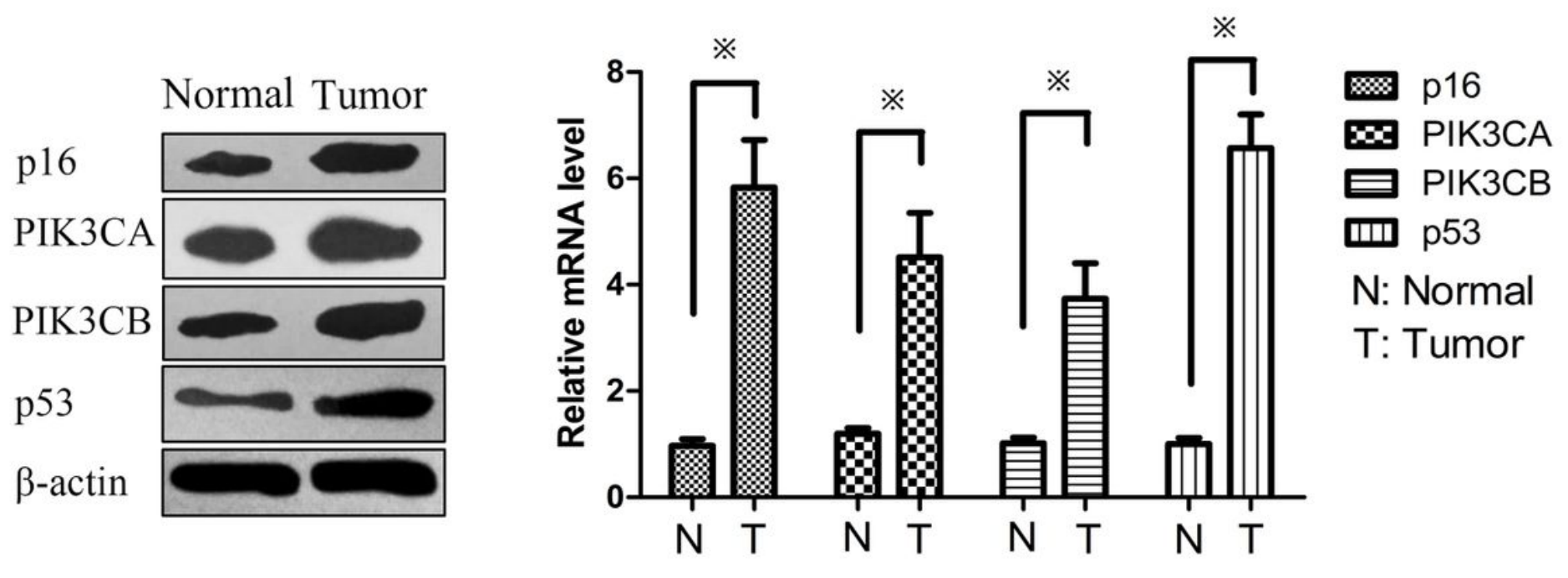

Figure 2

Western-blotting and Real-time-PCR analysis showed that the protein expression of p16, PIK3CA, PIK3CB and p53 in human esophageal squamous cell carcinomas tissues in comparison with non-tumor tissues have more upregulated expression. 

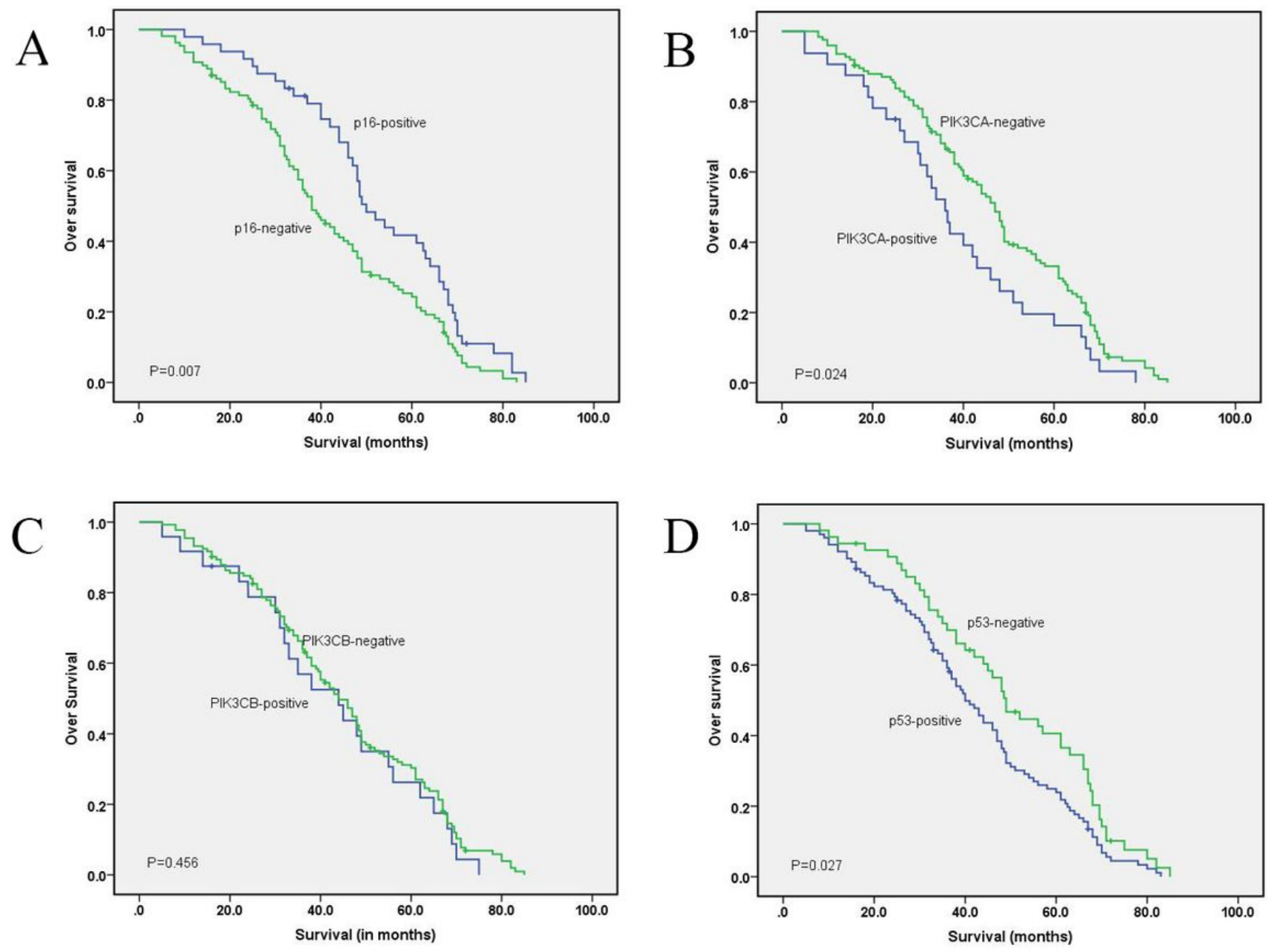

\section{Figure 3}

Kaplan-Meier survival curves for p16 (A), PIK3CA (B), PIK3CB (C) and p53 (D) expression in ESCC. In Kaplan-Meier analysis, high p16 expression correlated with a longer OS in ESCC patients than the corresponding controls. High PIK3CA and p53 expressions correlated with a shorter OS in ESCC patients than the corresponding controls. 